\title{
Sensitivity study of Protvino to ORCA (P2O) experiment: effect of antineutrino run, background and systematics
}

\author{
Sandhya Choubey ${ }^{\mathrm{a}}$, Monojit Ghosh ${ }^{\mathrm{b}}$, Dipyaman Pramanik ${ }^{\mathrm{c}}$ (B) \\ Harish-Chandra Research Institute, HBNI, Chhatnag Road, Jhunsi, Allahabad 211 019, India
}

Received: 19 December 2018 / Accepted: 23 June 2019 / Published online: 17 July 2019

(C) The Author(s) 2019

\begin{abstract}
There is a proposal to send a neutrino beam from the Protvino accelerator complex located in Russia to the detector facility called 'Oscillation Research with Cosmics in the Abyss' (ORCA) in the Mediterranean sea to study neutrino oscillation. This is called the $\mathrm{P} 2 \mathrm{O}$ experiment which will have a baseline of $2588 \mathrm{~km}$. In this paper we carry out an sensitivity study to extract the best possible physics sensitivity of the P2O experiment. In particular, we study the effect of antineutrino runs, the role of background as well as the impact of controlling the systematic uncertainties vis-a-vis the statistics.
\end{abstract}

\section{Introduction}

Ambitious and expensive next-generation long-baseline experiments are being proposed to measure the remaining crucial ingredients of the neutrino mass matrix - (i) the sign of $\Delta m_{31}^{2} a k a$ the neutrino mass hierarchy, (ii) the octant of $\theta_{23}$ and (iii) CP violation. The Deep Underground Neutrino Experiment (DUNE) [1] has been proposed to be built in USA, Tokai to Hyper-Kamiokande (T2HK) [2] proposal has been made for a long-baseline experiment in Japan and $\mathrm{ESS} \nu \mathrm{SB}$ [3] has been put forth as a prospective long-baseline endeavour in Sweden. The detector technology for DUNE will be liquid argon time projection chamber, while for T2HK and ESS $\nu$ SB water Čerenkov detector will be used. The CP sensitivity of all these three proposals is very high. DUNE is designed to measure $\mathrm{CP}$ violation at around $5 \sigma$ C.L. with

\footnotetext{
a e-mail: sandhya@hri.res.in

be-mail: ghoshmonojit@hri.res.in

c e-mail: dipyamanpramanik@hri.res.in
}

its proposed $3+3$ years run for maximal $\mathrm{CP}$ violation. ${ }^{1}$ T2HK should be able to discover CP violation at $7 \sigma$ C.L. in $5+5$ years (assuming that the mass hierarchy is known), while $\mathrm{ESS} \nu \mathrm{SB}$ 's projected sensitivity to $\mathrm{CP}$ violation is $8 \sigma$ from a $5+5$ years run. The octant sensitivity at these experiments is also seen to be very good with DUNE, T2HK and $\mathrm{ESS} \nu \mathrm{SB}$ promising a $3 \sigma$ discovery for $\left(\theta_{23}<43.5^{\circ}\right.$ and $\left.\theta_{23}>47.5^{\circ}\right),\left(\theta_{23}<43^{\circ}\right.$ and $\left.\theta_{23}>48^{\circ}\right)$ and $\left(\theta_{23}<41^{\circ}\right.$ and $\theta_{23}>50^{\circ}$ ), respectively. On the other hand, the hierarchy sensitivity is good only in DUNE where we can expect a $15 \sigma(7.5 \sigma)$ discovery for $\delta_{C P}=-90^{\circ}\left(+90^{\circ}\right)^{2}$ after a total run-time of 10 years. The ESSvSB set-up is not expected to have any hierarchy sensitivity at all, while for T2HK little hierarchy sensitivity is expected if both the water tanks are placed in Japan at a distance of $295 \mathrm{~km}$. In order to alleviate this problem to some extent, there is a proposal to put the second tank of the Hyper-Kamiokande detector in Korea, at a distance of about $1100 \mathrm{~km}$ from Tokai [2], bringing in matter effects and hence some hierarchy sensitivity. This proposal is called Tokai to Hyper-Kamiokande Korea (T2HKK) [2]. The hierarchy sensitivity for T2HKK proposal though is expected to be not significantly above $5 \sigma$ C.L. even after 5 +5 years of run time. Note that all the projected sensitivity reaches mentioned above are assuming $\mathrm{NH}$ to be true. For $\mathrm{IH}$ the sensitivities of all experiments are expected to be lower, especially for mass hierarchy. For studies regarding measuring the unknowns in the standard three flavour framework in these future long-baseline experiments see Refs. [4-18].

The main reason for lower hierarchy sensitivity in the above mentioned next-generation long-baseline experiments is their shorter baseline. To keep the experiment at the oscillation maximum the corresponding fluxes are made to peak at lower energies such that $L / E$ remains constant. The mat-

\footnotetext{
${ }^{1}$ By $x+y$ we imply running the experiment for $x$ years in the neutrino mode and $y$ years in antineutrino mode.

2 Throughout the paper we use the acronyms NH for normal hierarchy and IH for inverted hierarchy.
} 
ter potential of the earth is proportional to the energy of the neutrino. Therefore the matter potential is smaller for the shorter baseline experiments and thus the hierarchy sensitivity is smaller in these experiments. To overcome this, there is another European experimental proposal with a relatively longer baseline. This proposal is known as the Protvino to Oscillation Research with Cosmics in the Abyss (ORCA) or the P2O experiment [19-23]. This proposal comprises of shooting a neutrino beam from the Protvino accelerator site in Russia to the ORCA detector in the Mediterranean sea at a distance of $2588 \mathrm{~km}$ from the accelerator. The Protvino accelerator site currently houses the U-70 accelerator. The proposal is to upgrade the accelerator with an increased beam power of $450 \mathrm{KW}$ to deliver $4 \times 10^{20}$ protons on target per year. These protons are collided with a target to produce pions. These pions then go through a focusing system and decay pipe to produce a neutrino beam which is peaked at about $5 \mathrm{GeV}$, in order to coincide with the oscillation maximum for this baseline [24]. This larger beam energy brings in significant matter effects in the oscillation probability giving $\mathrm{P} 2 \mathrm{O}$ a clear edge over the other next-generation experiments in measuring the neutrino mass hierarchy. We will see that even with just 1 year of running of this experiment, one would measure the neutrino mass hierarchy at more than $3.5 \sigma$ in the worst possible scenario and greater than $5 \sigma$ in the best possible case. This extremely high sensitivity of $\mathrm{P} 2 \mathrm{O}$ to neutrino mass hierarchy comes also from the megaton-scale mass of the ORCA detector as well as the fact that the $2588 \mathrm{~km}$ baseline is very close to being bi-magic $[25,26] .^{3}$

In this paper we make a detailed study on the sensitivity of the experimental set-up for measuring the three unknowns in neutrino physics mentioned above. We will look into how the detector systematics and detector backgrounds affect the $\mathrm{CP}$ violation and octant of $\theta_{23}$ sensitivities of this experiment vis$\mathrm{a}$-vis the number of years of running of the experiment. We find that the hierarchy sensitivity of $\mathrm{P} 2 \mathrm{O}$, especially for $\mathrm{NH}$ true, is so high that any deterioration coming from uncontrolled backgrounds and/or systematic uncertainties makes no practical difference and one is ensured a sure shot discovery of the neutrino mass hierarchy within a very short time. The impact of systematics and backgrounds show up when the hierarchy is inverted, nonetheless a clear discovery of mass hierarchy is guaranteed, albeit with slightly higher run time than needed for the $\mathrm{NH}$ true case. We will show that the time needed for hierarchy discovery at P2O even with IH true will be significantly shorter than that needed at other competing experiments. On the other hand, the $\mathrm{CP}$ violation and octant sensitivity of $\mathrm{P} 2 \mathrm{O}$ is seen to be weaker than the corresponding expected sensitivity at competing long-

\footnotetext{
3 At bi-magic basline $(L=2540 \mathrm{~km})$, there is no $\delta_{C P}$ dependence in $\mathrm{NH}$ (IH) for 1.9 (3.3) GeV. This ensures hierarchy measurement without any $\delta_{C P}$ degeneracy with a high confidence level.
}

baseline experiments. We will show that these sensitivities can be improved mildly to severely with changes in systematic uncertainties and background. Also, for all cases, we study the impact of adding the antineutrino run to the projected sensitivity at $\mathrm{P} 2 \mathrm{O}$. Note that the $\mathrm{P} 2 \mathrm{O}$ collaboration in their first results [19] has shown the sensitivity to hierarchy and $\mathrm{CP}$ violation using 3 years run in the neutrino channel only. We find that a small antineutrino run fraction helps in getting rid of parameter degeneracies and helps in improving the expected sensitivity of $\mathrm{CP}$ violation and octant of $\theta_{23}$. We will quantify each of these aspects in what follows.

The rest of the paper is organised in the following way. In Sect. 2 we spell out our experimental and simulation details. In Sect. 3 we present the main results of our paper assuming $\mathrm{NH}$ to be true. In Sect. 4 we present our main findings on what happens when the true hierarchy is inverted instead of normal. Finally in Sect. 5 we compare the sensitivity of the P2O experiment with the other future long-baseline experiments. We conclude in Sect. 6.

\section{Experimental and simulation details}

As mentioned above, the U-70 accelerator located at the Protvino accelerator complex $100 \mathrm{~km}$ south of Moscow, is proposed to be upgraded to have $450 \mathrm{KW}$ beam power to deliver $4 \times 10^{20}$ protons on target (pot) per year. The neutrinos produced in this accelerator will be detected at ORCA at a distance of $2588 \mathrm{~km}$. At this baseline the first oscillation maximum occurs at $5 \mathrm{GeV}$ in vacuum. To simulate the $\mathrm{P} 2 \mathrm{O}$ experiment we follow the experimental details given in Ref. [19]. ${ }^{4}$ Our first aim is to obtain the event rates $N$. We take $1 \mathrm{GeV}$ bins in the energy range $1.5-10 \mathrm{GeV}$ and calculated the events at the far detector with the GLoBES [27] software using the following formula:

$$
N=\iint_{d E_{t} d E_{m}} \Phi\left(E_{t}\right) P_{\alpha \beta}\left(x, E_{t}\right) R\left(E_{t}, E_{m}\right) \sigma\left(E_{t}\right) M\left(E_{t}\right) \epsilon\left(E_{t}\right)
$$

where $\Phi$ is the neutrino flux $E_{t}$ is the true energy, $E_{m}$ is the reconstructed energy, $P_{\alpha \beta}$ is neutrino oscillation probability for $v_{\alpha} \rightarrow v_{\beta}$ with $\alpha, \beta=\mathrm{e}, \mu$ and $\tau, x$ are the neutrino oscillation parameters, $R$ is the energy resolution function, $\sigma$ is the cross section, $M\left(E_{t}\right)$ is the detector mass and $\epsilon\left(E_{t}\right)$ is the efficiency factor. To calculate $N$ we used the following information: (i) both the neutrino and antineutrino fluxes are obtained from the $\mathrm{P} 2 \mathrm{O}$ collaboration, (ii) the effective mass is taken from slide 8 of Ref. [19], (iii) the energy resolution is taken to be Gaussian with $30 \%$ width as given in slide 24 of

\footnotetext{
${ }^{4}$ Note that while our paper was under review, a detailed analysis by the $\mathrm{P} 2 \mathrm{O}$ collaboration appeared on arXiv [23] which mentioned that the results found are comparable to ours.
} 
Fig. 1 Events spectrum vs energy. Numbers are generated at $\theta_{12}=33.4^{\circ}, \theta_{13}=8.42^{\circ}$, $\Delta m_{21}^{2}=7.53 \times 10^{-5} \mathrm{eV}^{2}$ and $\Delta m_{31}^{2}= \pm 2.44 \times 10^{-3} \mathrm{eV}^{2}$. The solid (dashed) purple/blue/green/red curves correspond to muon (electron) events for $\delta_{C P}=0^{\circ} / 90^{\circ} / 180^{\circ} / 270^{\circ}$. These events corresponds to 3 years running of $\mathrm{P} 2 \mathrm{O}$ in the neutrino mode corresponding to $12 \times 10^{20}$ proton on target
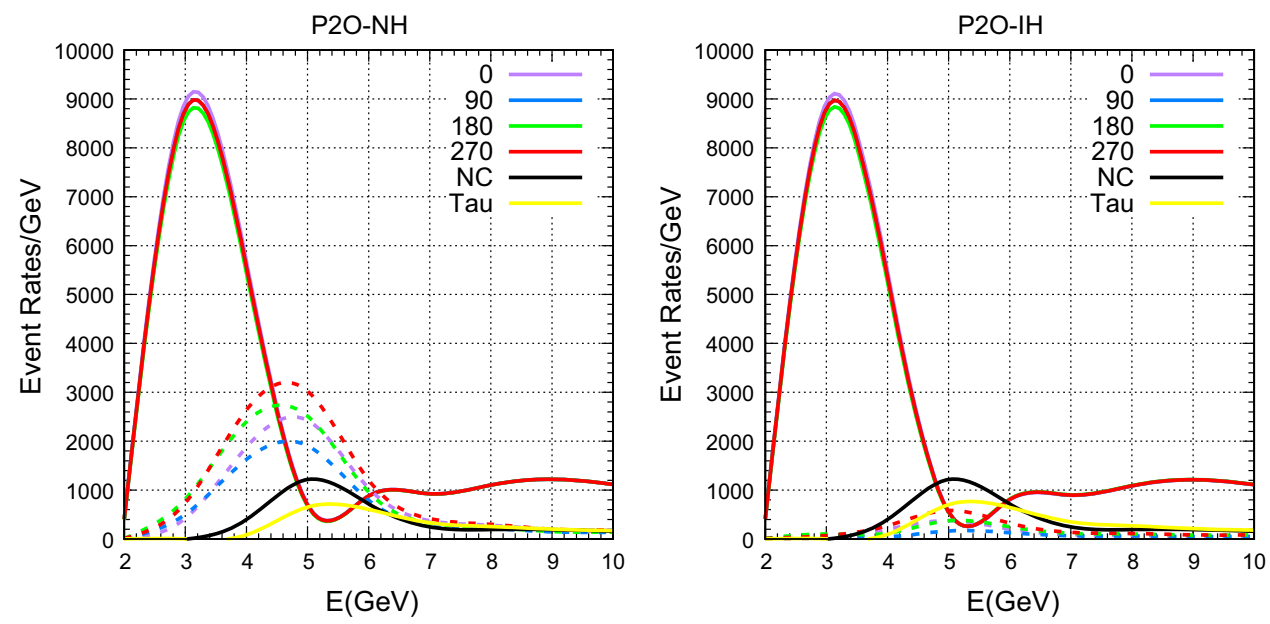
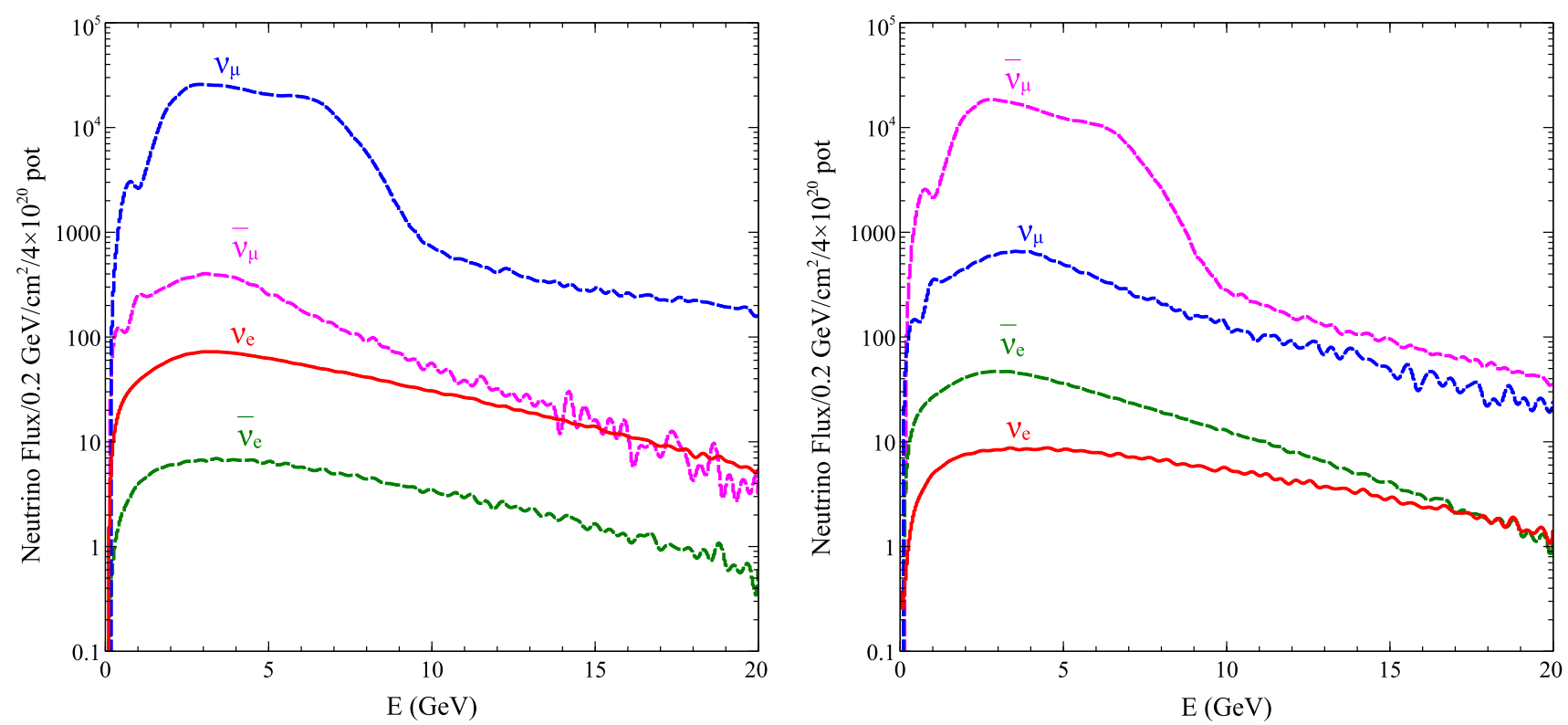

Fig. 2 Fluxes as a function of energy. The left panel is for neutrino flux and the right panel is for antineutrino flux

[19]. Finally we implemented energy dependent efficiencies to match with the event spectrum in normal hierarchy as presented in slide 9 of [19]. We have given in the left panel of Fig. 1. Then we generate events for IH taking the same specification which we used for normal hierarchy and this perfectly matches with the event spectrum as given in slide 10 of Ref. [19]. This we have given in the right panel of Fig. 1. The choice of neutrino oscillation parameters are same as in slide 11 of [19]. These events corresponds to 3 years running of the $\mathrm{P} 2 \mathrm{O}$ experiment in the neutrino mode corresponding ot $12 \times 10^{20}$ proton on target. ${ }^{5} \mathrm{We}$ have presented the energy dependence of the fluxes in Fig. 2. The left (right) panel is

\footnotetext{
${ }^{5}$ Note our method of calculating events is simplified and does not match-up with the sophistication that the analysis of the experimental collaboration would have. But nevertheless with this calculation of events, we successfully reproduce the sensitivity results of $\mathrm{P} 2 \mathrm{O}$.
}

for neutrinos (antineutrinos). In the panels we have showed all the four components of the neutrino/antineutrino fluxes. The muon neutrino/antineutrino fluxes mainly play the major role in the sensitivity of $\mathrm{P} 2 \mathrm{O}$. After matching the events our next aim is to match the $\chi^{2}$. But before that lets discuss the event topologies of the $\mathrm{P} 2 \mathrm{O}$ experiment.

The two main event topologies in ORCA are track and cascade. The tracks are almost always produced by $v_{\mu}$ charged current interactions while cascades come from $v_{e}$ charged current interactions, $\nu_{\tau}$ charged current interactions as well as neutral current interactions of neutrinos. In our simulations we have simulated track and cascade events for both neutrino and antineutrino channels by suitably modifying GLoBES. A brief discussion on the backgrounds follows. Since the tracks come from $v_{\mu}$ charged current interactions alone, the only backgrounds for the disappearance channel come from neu- 
tral current backgrounds ${ }^{6}$ and tau backgrounds. For appearance channel, backgrounds come from $v_{\tau}$ charged current interactions and neutral current interactions as well as misidentification ${ }^{7}$ of the muon events as electron events. The numbers for tau and neutral current background which we will use in our analysis are same as given in Fig. 1.

For the fit method, we use the following informations: (i) we have considered both electron (appearance) and muon (disappearance) events, (ii) apart from the backgrounds mentioned above we have taken additional $20 \%$ mis-id background in the appearance channel. Note that when we implement the mis-id background for appearance channel, we subtract the same mis-id factor from the disappearance channel signal events. For systematics we follow slide 11 of Ref. [19]. We have taken an overall normalization error of 5\% for both appearance and disappearance channel signal. The background systematic error for disappearance channel is calculated by adding the normalization error for tau (10\%) and normalization error for neutral current $(5 \%)$ in quadrature which is $11 \%$. For appearance channel background we have taken $6 \%$ error. For the neutrino oscillation parameters we have taken the values as given in slide 11 of Ref. [19]. With this specification we reproduce the $\chi^{2}$ plots of [19]. These plots are hierarchy sensitivity curve vs $\theta_{23}$, CP violation sensitivity curve vs $\delta_{C P}, \mathrm{CP}$ precision curves vs $\delta_{C P}$ and $\delta_{C P}$ resolution curve vs running time as given in slide numbers $12,13,14$ and 15 of [19] respectively. Following [19], we have not considered any systematic error to incorporate a shift in the energy scale. Note that Ref. [19] does not give any information on antineutrinos, therefore we take the exact same values of the efficiencies, background and systematics for the antineutrino analysis.

In the following sections we will discuss the hierarchy, octant and $\mathrm{CP}$ sensitivity of the P2O experiment. As the present data hints the preference of $\mathrm{NH}$ over $\mathrm{IH}$, we present our results for $\mathrm{NH}$ in detail in the next section. After that we will give the results for IH for some selective values of parameters.

\section{Results for NH}

In this section we discuss the capability of the $\mathrm{P} 2 \mathrm{O}$ experiment to discover the unknowns in the three flavour standard neutrino oscillations, namely neutrino mass hierarchy, octant of the mixing angle $\theta_{23}$ and measurement of the Dirac type phase $\delta_{C P}$. While calculating $\chi^{2}$ we have taken both the

\footnotetext{
${ }^{6}$ Note that though NC background is shower like, some of them can be confused with muon track events and is hence one of the indispensable backgrounds of the muon track events. However, this background does not play much role in the analysis of track events.

${ }^{7}$ We will refer to this background as mis-id throughout our paper.
}

electron and muon events. Our aim of this study will be to find out the optimal configuration of the $\mathrm{P} 2 \mathrm{O}$ experiment to discover the above mentioned parameters by considering various combinations of antineutrino exposure, systematic errors and backgrounds. For systematic uncertainty, we only vary the error associated with appearance channel signal and the only background we vary is the mis-id in the appearance channel. In our $\chi^{2}$ analysis we have kept fixed the parameters $\theta_{12}=33.4^{\circ}, \theta_{13}=8.42^{\circ}, \Delta m_{21}^{2}=7.53 \times 10^{-5} \mathrm{eV}^{2}$ and $\Delta m_{31}^{2}=2.44 \times 10^{-3} \mathrm{eV}^{2}$ in both the true and test spectrum. These values are consistent with the global analysis of the world neutrino data [28-30].

\subsection{Hierarchy}

The hierarchy sensitivity of an experiment is calculated by taking the correct hierarchy in the true spectrum and the wrong hierarchy in the test spectrum. First we study the effect of antineutrino run on the hierarchy sensitivity of the $\mathrm{P} 2 \mathrm{O}$ experiment.

In Fig. 3 we have plotted the hierarchy $\chi^{2}$ vs $\delta_{C P}$ (true). The parameters $\theta_{23}$ and $\delta_{C P}$ have been marginalized in the test. The left/middle/right panel is for $\theta_{23}$ (true) value of $42^{\circ} / 45^{\circ} / 48^{\circ}$. In these plots we have assumed a total run-time of 6 years and we have considered four different combinations of neutrino and antineutrino ratio.

In all the three panels we see that the hierarchy sensitivity is maximum at $\delta_{C P}=-90^{\circ}$ and minimum at $\delta_{C P}=+90^{\circ}$. This is because of the well known hierarchy $\delta_{C P}$ degeneracy $[31,32]$. In the case of $\mathrm{P} 2 \mathrm{O}$, this degeneracy is lifted because of the large matter effect. But still the probability in $\mathrm{NH}$ corresponding to $\delta_{C P}=-90^{\circ}\left(+90^{\circ}\right)$ is furthest (closest) to the probability in the IH. This is true for both neutrino and antineutrino. To understand the role of antineutrinos we need to understand the behavior of octant degeneracy. It was shown in $[33,34]$ that the main role of the antineutrino run is to resolve the octant degeneracy to improve the $\mathrm{CP}$ and hierarchy sensitivity in the long-baseline experiments. Therefore, if the wrong hierarchy $\chi^{2}$ occurs with the wrong octant, then addition of antineutrinos can improve the hierarchy $\chi^{2}$ by removing the wrong octant solution. That is what is happening for $\theta_{23}=42^{\circ}$ (left panel). For $6+0$ years, the hierarchy $\chi^{2}$ occurs with the wrong octant and $5+1$ years gives better sensitivity than $6+0$ years. In this panel we also note that further addition of antineutrinos decreases the sensitivity. From this we understand that only 1 year of antineutrino is sufficient to remove the octant degeneracy and further addition of antineutrino only decreases the statistics due its low cross section and smaller flux as compared to the neutrinos. The situation is slightly different for $\theta_{23}=48^{\circ}$ (right panel). At this true value of $\theta_{23}$ the hierarchy $\chi^{2}$ appears with the right octant and thus antineutrino should not help much. That is why we see that the sensitivity is almost best for $6+0$ years for 

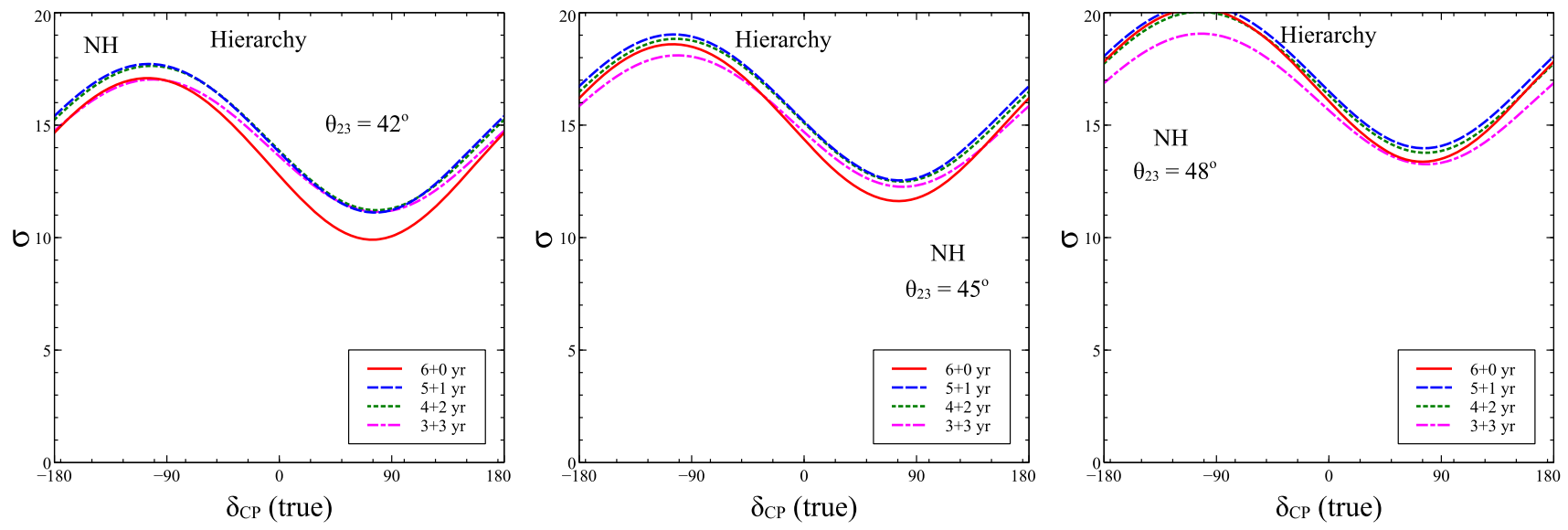

Fig. 3 Hierarchy sensitivity vs $\delta_{C P}$ (true) in NH for different combinations of neutrino and antineutrino ratio. The left/middle/right panel is for $\theta_{23}($ true $)=42^{\circ} / 45^{\circ} / 48^{\circ}$

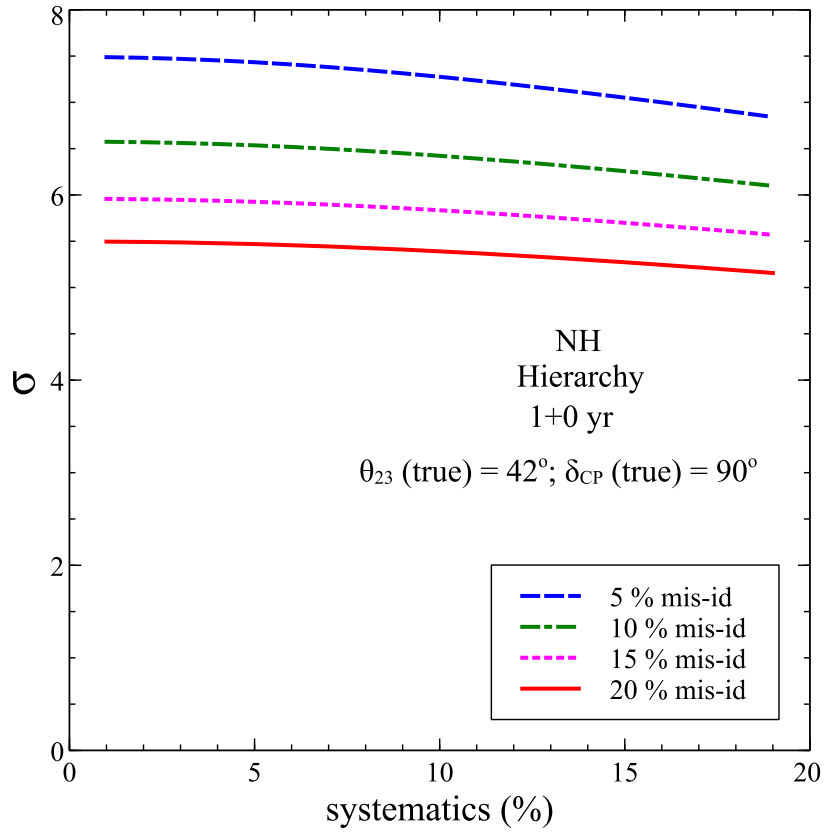

Fig. 4 Effect of background and systematics in hierarchy sensitivity for NH. This is shown for $\theta_{23}$ (true) $=42^{\circ}$ and $\delta_{C P}$ (true) $=90^{\circ}$ as for this set of parameters the $\chi^{2}$ is minimum in $\mathrm{NH}$

$\delta_{C P}=-90^{\circ}$ (true). However the best sensitivity is obtained for $5+1$ years. Now let us discuss for $\theta_{23}=45^{\circ}$ where there is no octant degeneracy (middle panel). Here we see that at $\delta_{C P}$ (true) $=-90^{\circ}, 5+1$ years give best sensitivity and with further addition of antineutrinos, sensitivity decreases. Whereas for $\delta_{C P}$ (true) $=90^{\circ}, 5+1$ years gives the best sensitivity and the worst sensitivity comes for $3+3$ years and 6 +0 years.

From these three panels we see that the minimum hierarchy sensitivity for 6 year running of the experiment is always greater than $10 \sigma$, irrespective of the antineutrino exposure.
Next we study the effect of background and systematics on hierarchy sensitivity. In Fig. 4, we have presented the hierarchy $\chi^{2}$ vs systematic uncertainty for the four combinations of background. We present this for just 1 year of neutrino exposure of the $\mathrm{P} 2 \mathrm{O}$ experiment and for $\delta_{C P}($ true $)=+90^{\circ}$ and $\theta_{23}$ (true) $=42^{\circ}$. As the $\chi^{2}$ is minimum for this value of $\delta_{C P}$ and $\mathrm{NH}$ true, this gives the most conservative configuration of the experiment. From this plot we understand that the sensitivity does not vary much with the systematic uncertainty. For all the four combinations of the background, hierarchy sensitivity falls only within $1 \sigma$ when systematic is varied from 1 to $20 \%$. On the other hand we observe that the sensitivity depends greatly on the amount of background. For 5\% systematic error, the sensitivity goes from $5.5 \sigma$ to $7.5 \sigma$ when background decreases from 20 to $5 \%$. However, it is very important to note from this plot that whatever be the systematic error or the background, $\mathrm{P} 2 \mathrm{O}$ has hierarchy sensitivity always more than $5 \sigma$, even with 1 year neutrino run. This can be attributed to two facts: (i) huge matter effect enable $\mathrm{P} 2 \mathrm{O}$ to have a very large sensitivity and (ii) the baseline for P2O is close to the bi-magic baseline $[25,26]$ for which the hierarchy sensitivity gets enhanced at a particular energy.

\subsection{Octant}

Octant sensitivity of an experiment is calculated by taking the correct octant in the true spectrum and wrong octant in the test spectrum. We first study the effect of antineutrinos in the determination of the octant. In Fig. 5, we have presented the octant sensitivity for different combinations of neutrino and antineutrino ratio, taking the total run-time of 6 years. In the left and middle panel we have presented the results as a function of $\delta_{C P}$ (true) and in the right panel we have given our results as a function of $\theta_{23}$ (true).The left and middle panel are for $\theta_{23}$ in the lower octant $\left(\theta_{23}=42^{\circ}\right)$ and higher 

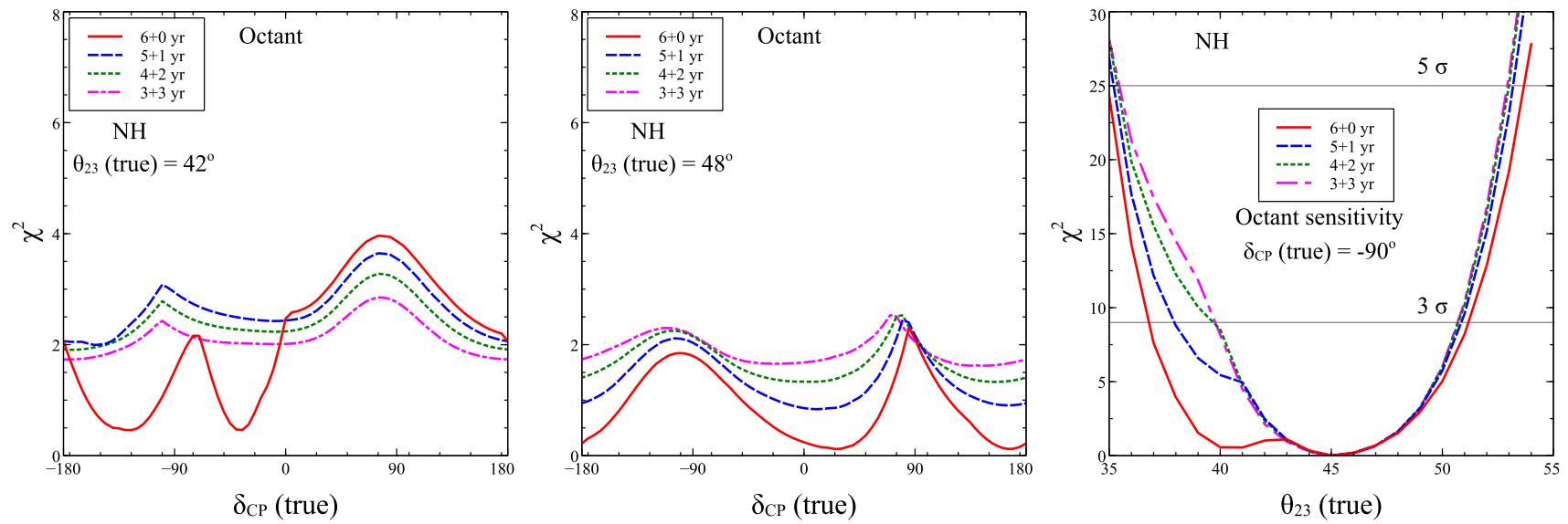

Fig. 5 Octant sensitivity in $\mathrm{NH}$ for different combinations of neutrino and antineutrino ratio. Octant $\chi^{2}$ vs $\delta_{C P}($ true) is presented in the left and middle panel for $\theta_{23}=42^{\circ}$ and $48^{\circ}$ respectively. Octant $\chi^{2}$ vs $\theta_{23}$ (true) is presented in the right panel for $\delta_{C P}=-90^{\circ}$

octant $\left(\theta_{23}=48^{\circ}\right)$, respectively. In the right panel, true value of $\delta_{C P}=-90^{\circ}$ which is the present best-fit value of this parameter. In all the panels $\theta_{23}$ has been marginalized in the opposite octant and $\delta_{C P}$ has been marginalized in its full range. Hierarchy is also marginalized.

To understand the role of antineutrinos in resolving octant degeneracy, first we have to understand how octant degeneracy occurs in neutrinos and antineutrinos. It has been shown in $[32,35]$ that for the neutrino channel octant degeneracy occurs at $\left(-180^{\circ}<\delta_{C P}<0^{\circ}\right.$, LO $)$ with $\left(0^{\circ}<\delta_{C P}<180^{\circ}\right.$, $\mathrm{HO})$ and for the antineutrino channel octant degeneracy occurs at $\left(0^{\circ}<\delta_{C P}<180^{\circ}\right.$, LO $)$ with $\left(-180^{\circ}<\delta_{C P}<0^{\circ}\right.$, $\mathrm{HO})$. This is the reason why a balanced antineutrino run is always important to have significant octant sensitivity. The above discussion explains why for $42^{\circ}$ octant sensitivity is maximum for $6+0$ years for $0^{\circ}<\delta_{C P}<+180^{\circ}$ and minimum for $-180^{\circ}<\delta_{C P}<0^{\circ}$ (left panel). With the addition of an antineutrino run the sensitivity for $\delta_{C P}=+90^{\circ}$ decreases and sensitivity for $\delta_{C P}=-90^{\circ}$ increases. From the plot we also realise that the $5+1$ years is an optimal choice of exposure. For this exposure, the octant sensitivity of $\chi^{2}=3.5(4)$ can be obtained for $\delta_{C P}=-90^{\circ}\left(+90^{\circ}\right)$. For $\theta_{23}=48^{\circ}$ we notice that the sensitivity is maximum for $3+3$ years at $\delta_{C P}=-90^{\circ}$ and minimum for $6+0$ years. Whereas for $\delta_{C P}=+90^{\circ}$ all the combinations of the antineutrino run give almost equal sensitivity. Note that the sensitivity in the higher octant is smaller than the sensitivity in the lower octant. This is due to the fact that for the higher octant, the denominator in the $\chi^{2}$ is higher than the denominator in the $\chi^{2}$ for lower octant. Here the octant sensitivity is around $\chi^{2}=2.5$ for both $\delta_{C P}=-90^{\circ}$ and $+90^{\circ}$. The right panel shows octant sensitivity for different values of $\theta_{23}$ for $\delta_{C P}=-90^{\circ}$. From the plot we see that in the lower octant, 6 +0 years gives the worst octant sensitivity and in the region $41^{\circ}<\theta_{23}<45^{\circ}, 5+1$ years give the best octant sensitivity. For higher octant all the four combination give almost equal sensitivity. From this plot we conclude that with the current background and systematics, $\mathrm{P} 2 \mathrm{O}$ can resolve octant at $3 \sigma$ for $\theta_{23}$ values except $41^{\circ}\left(39^{\circ}\right)<\theta_{23}<51^{\circ}$ for $3+3$ years $(5$ +1 years) run.

From the above discussion we realize that $5+1$ years would be the best combination for $\mathrm{P} 2 \mathrm{O}$ to determine the octant sensitivity for both LO and HO. But even for this best combination, a maximum $\chi^{2}$ of 4 can be achieved at the present best-fit of $\delta_{C P}$ and $\theta_{23}$ which is $-90^{\circ}$ and $42^{\circ} \&$ $48^{\circ}$. Next we study the effect of background and systematics in octant sensitivity with the neutrino to antineutrino ratio of 5:1.

In Fig. 6, we have presented the run-time to achieve a sensitivity of a certain confidence level as a function of background for different combination of systematic uncertainties. we present this for $\theta_{23}=42^{\circ}$ and $\delta_{C P}=-90^{\circ}$. From the figure we see that there is no effect of background and systematics to achieve $1 \sigma$ sensitivity as the required run-time remains almost fixed to almost 1 year for all the values of systematics and background. To achieve $2 \sigma$ sensitivity the run-time keeps increasing as we increase the systematics for a fixed value of background. For the most optimistic case (i.e., $0 \%$ systematic error), the required run-time increases from 4.5 to 7 years to achieve a $2 \sigma$ octant sensitivity when the background increases from 5 to $20 \%$.

\section{$3.3 \mathrm{CP}$}

First we discuss the $\mathrm{CP}$ violation (CPV) discovery potential of the $\mathrm{P} 2 \mathrm{O}$ experiment. $\mathrm{CP}$ violation discovery potential is defined as the capability to distinguish a value of $\delta_{C P}$ from the $\mathrm{CP}$ conserving values of $0^{\circ}$ and $180^{\circ}$. To study the role of antineutrinos, in Fig. 7, we have plotted sensitivities for four different combinations of neutrino to antineutrino ratio. The left $/$ middle/right panel is for $\theta_{23}=42^{\circ} / 45^{\circ} / 48^{\circ}$. In these figures we have marginalized over $\theta_{23}$ and hierarchy in the 


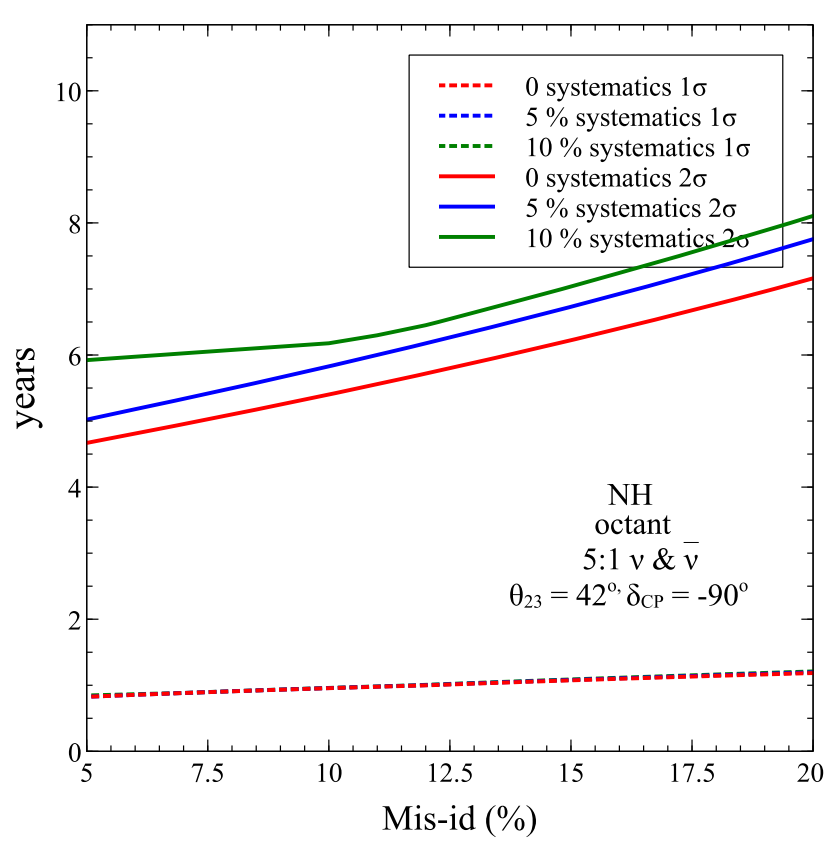

Fig. 6 Effect of background and systematics in octant sensitivity for $\mathrm{NH}$ in terms of runtime required to achieve a sensitivity of a certain confidence level. This is shown for $\theta_{23}($ true $)=42^{\circ}$ and $\delta_{C P}($ true $)=$ $-90^{\circ}$ as for this set of parameters are close to the present best-fit

test. The effect of octant degeneracy can be seen for $\left(\theta_{23}\right.$, $\left.\delta_{C P}\right)$ combination of $\left(42^{\circ},-90^{\circ}\right)$ and $\left(48^{\circ},+90^{\circ}\right)$ from the shape of the curves. This is because for the pure neutrino run (i.e., $6+0$ years) the $\mathrm{CPV} \chi^{2}$ occurs in the wrong octant for $\left(42^{\circ},-90^{\circ}\right)$ and $\left(48^{\circ},+90^{\circ}\right)$. Therefore the addition of an antineutrino run is supposed to help in the sensitivity by removing the octant degeneracy. From the panel we understand that this is exactly what is happening as the sensitivity keeps improving with the addition of antineutrino data. However from the shape of the curves it is also clear that even with the addition of antineutrino runs, the octant degeneracy is not completely removed. For the other two combinations i.e., $\left(42^{\circ},+90^{\circ}\right)$ and $\left(48^{\circ},=-90^{\circ}\right)$ we note that $6+0$ years and $5+1$ years give the best sensitivity, respectively. The middle panel shows the CPV sensitivity without any octant degeneracy. From this panel we note that addition of antineutrino run helps in improving the CPV sensitivity. For $\delta_{C P}=-90^{\circ}, 3$ +3 years give best sensitivity and for $\delta_{C P}=+90^{\circ}, 6+0$ years provide the best sensitivity. From the discussion we can also conclude that the antineutrino run is important for CPV discovery and $5+1$ years will be the best option for P2O. It is also important to note that irrespective of the combination of the antineutrino run, the CPV discovery is always less than $3 \sigma$ for all the three combinations of $\theta_{23}$.

We next focus on the effect of background and systematics in the CPV sensitivity of P2O. In Fig. 8 we have plotted the time required to achieve a CPV sensitivity of a certain confidence level vs the background for different combination of the systematic uncertainty. We have done this for $\theta_{23}=42^{\circ}$ and $\delta_{C P}=-90^{\circ}$. The curves are for equal ratios of neutrinos and antineutrinos. From the plot we see that to achieve $1 \sigma$ sensitivity, $\mathrm{P} 2 \mathrm{O}$ requires around 1 year of running, irrespective of the value of systematic and background. The effect of systematic and background comes into the picture to achieve a sensitivity at a higher confidence level. To achieve a $2 \sigma$ $\mathrm{CPV}$ discovery, $\mathrm{P} 2 \mathrm{O}$ will require around 3.5 years if the background is $5 \%$ and 5.5 years if the background is $20 \%$. This is true irrespective of the systematic error. From the figure we also note that the systematic uncertainty starts to play some role when $\mathrm{P} 2 \mathrm{O}$ tries to achieve a CPV sensitivity of $3 \sigma$ as the curves for different systematic errors tends to separate from each other. Here we observe that the required run-time of $\mathrm{P} 2 \mathrm{O}$ is more than 10 years to achieve a $3 \sigma \mathrm{CPV}$ discovery if the background is larger than $7.5 \%$.

At this point we want to stress the fact that although $\mathrm{P} 2 \mathrm{O}$ will achieve a $5 \sigma$ hierarchy sensitivity in less than 1 year, it can only achieve a $2 \sigma \mathrm{CPV}$ and octant sensitivity in 6 years
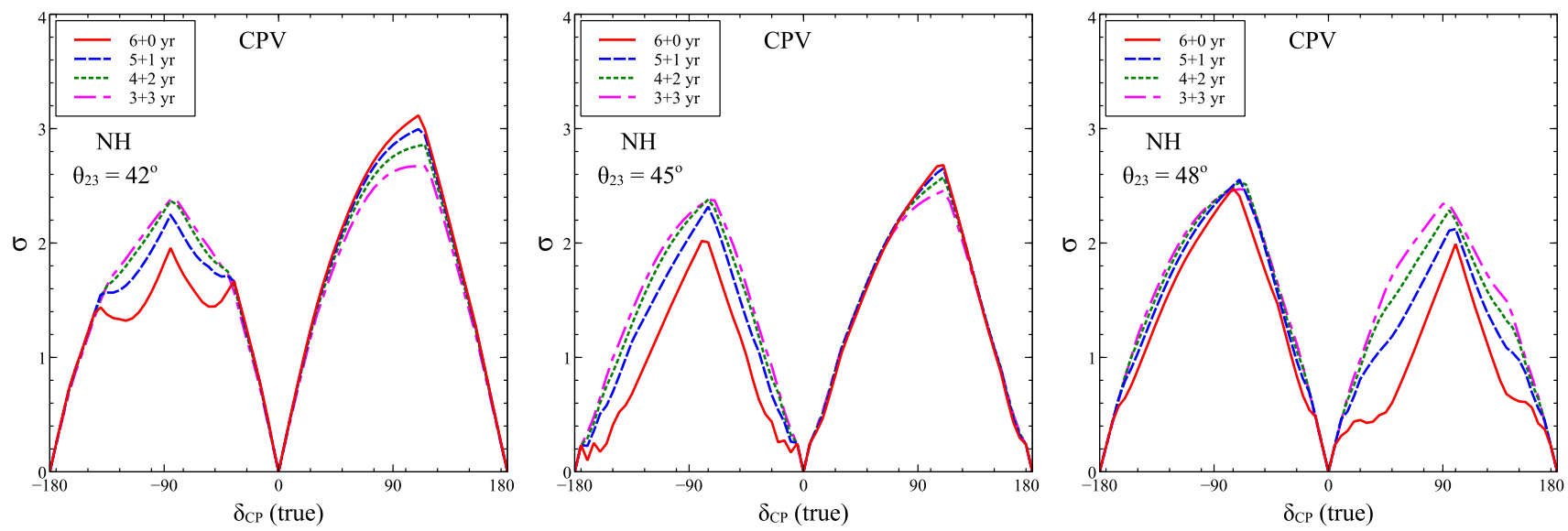

Fig. $7 \mathrm{CPV}$ discovery sensitivity vs $\delta_{C P}$ (true) for different combinations for neutrino and antineutrino in NH. The left/middle/right panel is for $\theta_{23}=42^{\circ} / 45^{\circ} / 48^{\circ}$ 


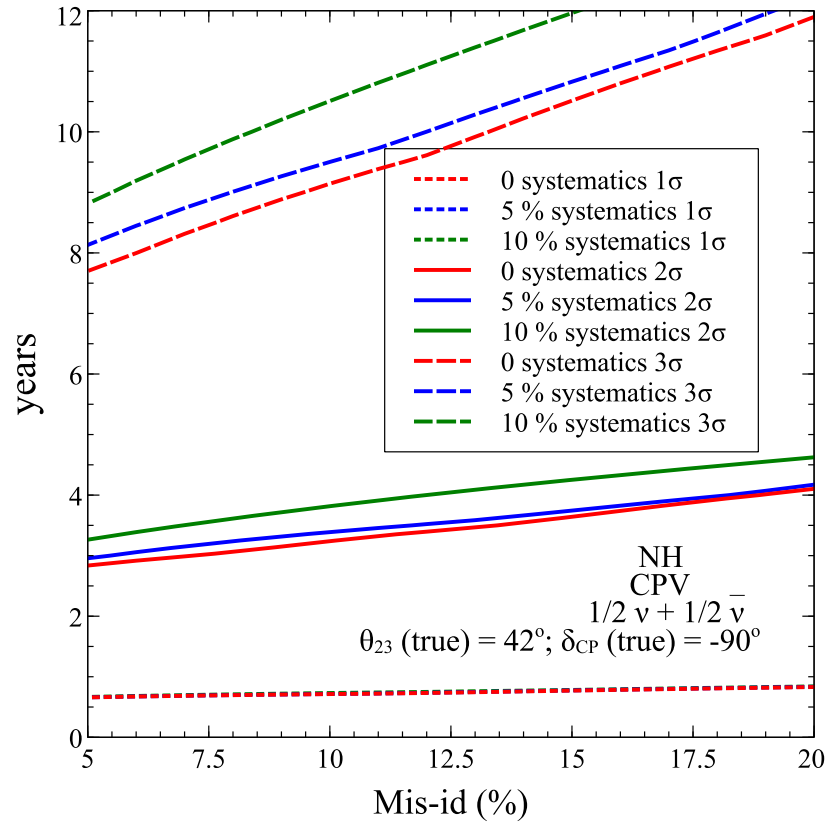

Fig. 8 Effect of background and systematics for CPV discovery in $\mathrm{NH}$. in terms of runtime required to achieve a sensitivity of a certain confidence level. This is shown for $\theta_{23}$ (true $)=42^{\circ}$ and $\delta_{C P}$ (true $)=$ $-90^{\circ}$ as for this set of parameters are close to the present best-fit

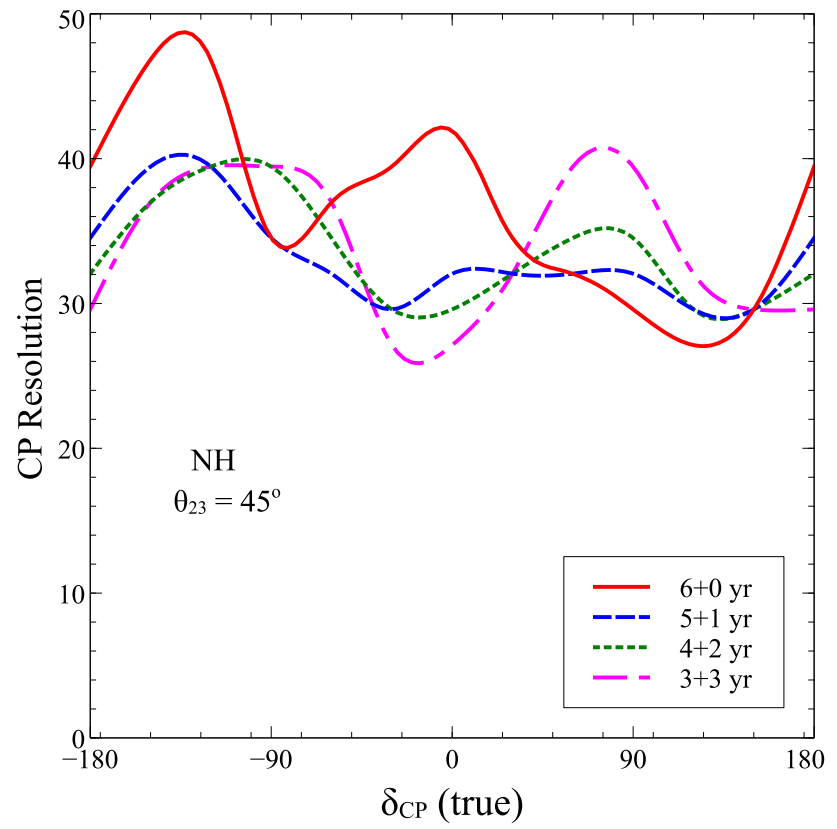

Fig. $9 \mathrm{CP}$ resolution sensitivity in $\mathrm{NH}$. This is shown for $\theta_{23}$ (true)= $45^{\circ}$ and taking four different combinations of neutrino-antineutrino ratio

of run-time. As we mentioned earlier the hierarchy sensitivity at this baseline is enhanced due to larger matter effect and bi-magic property. The lack of octant and CP sensitivity stems from the poor energy resolution and particle identification. One solution can be optimization using more dense instrumentation of the ORCA detector which can improve the energy resolution and particle identification.

Let us now turn to how precisely $\mathrm{P} 2 \mathrm{O}$ will be able to measure a value of $\delta_{C P}$. To study this, in Fig. 9 we have plotted the $\mathrm{CP}$ resolution for each value of true $\delta_{C P}$ for $\theta_{23}$ (true) $=45^{\circ}$. We define $\mathrm{CP}$ resolution by $0.5 \times$ allowed range of $\delta_{C P}$ values at $1 \sigma / 360$. In this figure we have marginalized over $\theta_{23}$ and hierarchy in the test. This we have presented for four combination of antineutrino runs. From the curves we see that the $\mathrm{CP}$ precision is best around $0^{\circ}$ and $180^{\circ}$ and worst around $\pm 90^{\circ}$. Apart from these we can also notice a few local mininma which are more prominent in the case of pure neutrino run and getting removed when antineutrino run is added. This is due to the parameter degeneracies which are present in the pure neutrino run and getting removed when antineutrino run is added. From this figure we understand that the best $\mathrm{CP}$ resolution is achieved for the $5+1$ years combination. For this combination a $\delta_{C P}$ can be measured within $34 / 32 / 32$ degrees uncertainty for $\delta_{C P}$ (true) $=-90^{\circ} / 0^{\circ} /+90^{\circ}$.

\section{Discussion for sensitivity in IH}

In this section we discuss the sensitivity of the $\mathrm{P} 2 \mathrm{O}$ experiment in IH. In Fig. 10 we present the hierarchy sensitivity for $\theta_{23}=45^{\circ}$. In the left panel, we have given the sensitivity vs $\delta_{C P}$ (true) for a total run-time of 6 years. Like the case of $\mathrm{NH}$, we have divided the run-time for different combinations of neutrino and anti-neutrino ratio. Apart from the four combinations given for the $\mathrm{NH}$ case, we have also put $2+4$ years, $1+5$ years and $0+6$ years run-times. We have included the dominating anti-neturino run-times for IH because matter effect is reversed for the inverted hierarchy case. Here we can see that indeed anti-neutrino dominating run-times $2+$ 4 years and $1+5$ years are better, however, for the case of 0 +6 years the sensitivity is again lower. This is because the statistics in the antineutrino channel is smaller compared to the statistics in the neutrino channel. In the right panel of Fig. 10 , we have shown the variation of the hierarchy sensitivity with respect to background and systematics for a run-time of $1+0$ year. We have done this for $\delta_{C P}=-90^{\circ}$ for which the sensitivity in IH is minimum. This will give the most conservative estimate. From this we note that the effect of systematics is stronger in $\mathrm{IH}$ as compared to $\mathrm{NH}$. This plot shows that to achieve a $5 \sigma$ sensitivity, the systematic error can be allowed to increase from 9 to $13 \%$ if the background decreases from 20 to $5 \%$.

Next let us discuss the octant sensitivity of the $\mathrm{P} 2 \mathrm{O}$ experiment in IH. The octant sensitivity of the $\mathrm{P} 2 \mathrm{O}$ experiment for $\mathrm{IH}$ is very poor. We have checked that for a total run-time of 6 years, the octant sensitivity in pure neutrino run (i.e., 6 +0 years) is negligible for both $\theta_{23}=42^{\circ}$ and $48^{\circ}$. This is because of the fact the neutrino probability is smaller in $\mathrm{IH}$ 

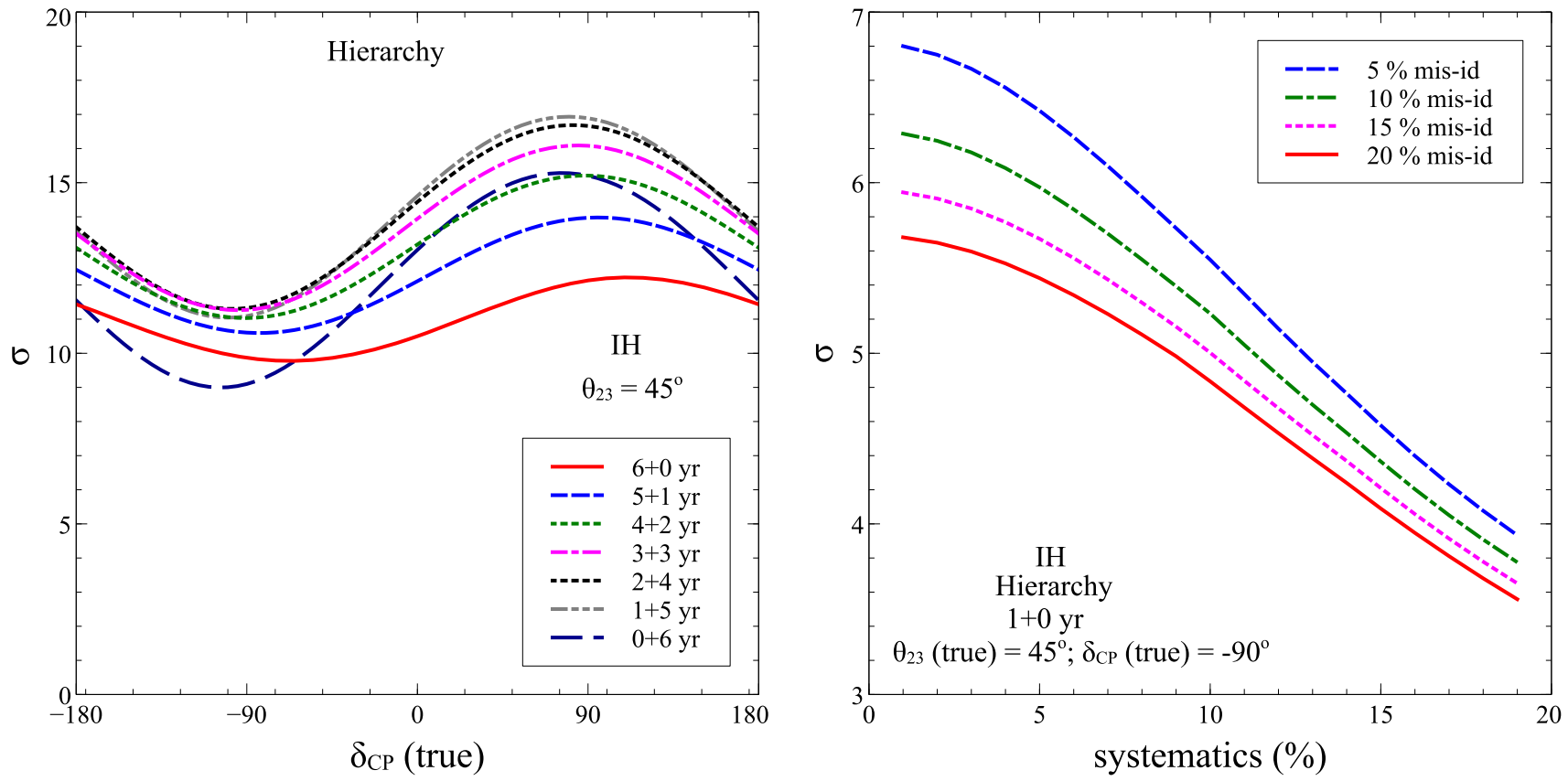

Fig. 10 Hierarchy sensitivity for different combinations for neutrino and antineutrino in IH is shown in the left panel for $\theta_{23}=45^{\circ}$. The right panel shows the impact of systematics and backgrounds on the hierarchy sensitivity in IH for $\theta_{23}=45^{\circ}$ and $\delta_{C P}=-90^{\circ}$
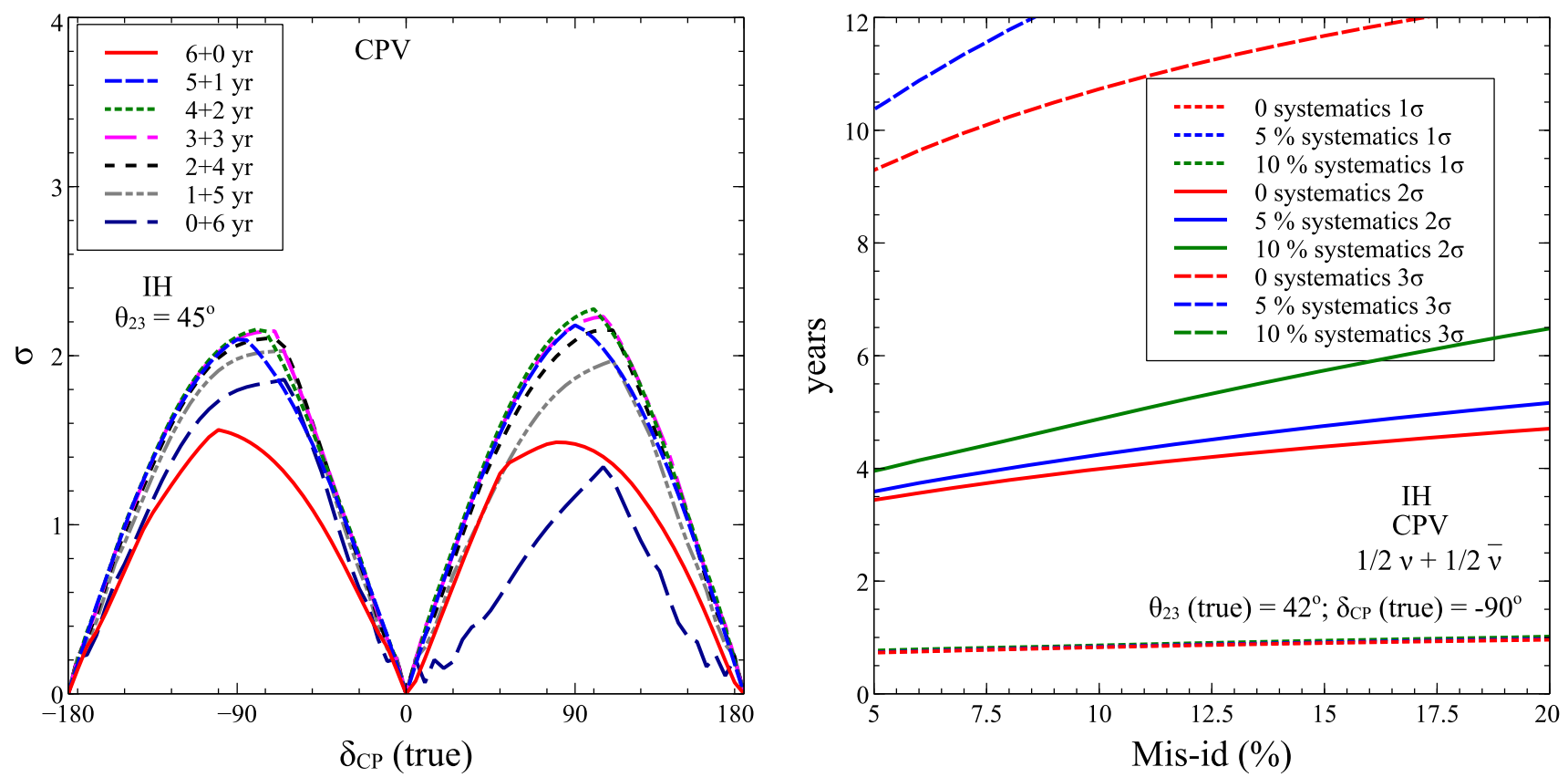

Fig. 11 The left panel shows the CPV sensitivity as a function of $\delta_{C P}$ (true) in IH for $\theta_{23}=45^{\circ}$. The right panel shows the impact of systematics and backgrounds on the CPV sensitivity in IH in terms of runtime required to achieve a sensitivity of a certain confidence level. This is for $\theta_{23}=45^{\circ}$ and $\delta_{C P}=-90^{\circ}$

for neutrinos as compared to NH. At both the above mentioned true values, the best octant sensitivity comes for $3+3$ years combination but the octant $\chi^{2}$ does not rise above 3 .

We have presented the results for CPV discovery potential of $\mathrm{P} 2 \mathrm{O}$ for different neutrino and antineutrino run-times for IH in Fig. 11 with a total run-time of 6 years and with $\theta_{23}=45^{\circ}$. In the left panel we have given the CPV discovery sensitivity as a function of $\delta_{C P}$ (true). As for the case of mass hierarchy, here also we have considered $2+4$ years, $1+5$ years and $0+6$ years of run-times along with $6+0$ years, $5+1$ years, $4+2$ years and $3+3$ years run-times. In the right panel of Fig. 11 we have given the required run-time to obtain a cer- 


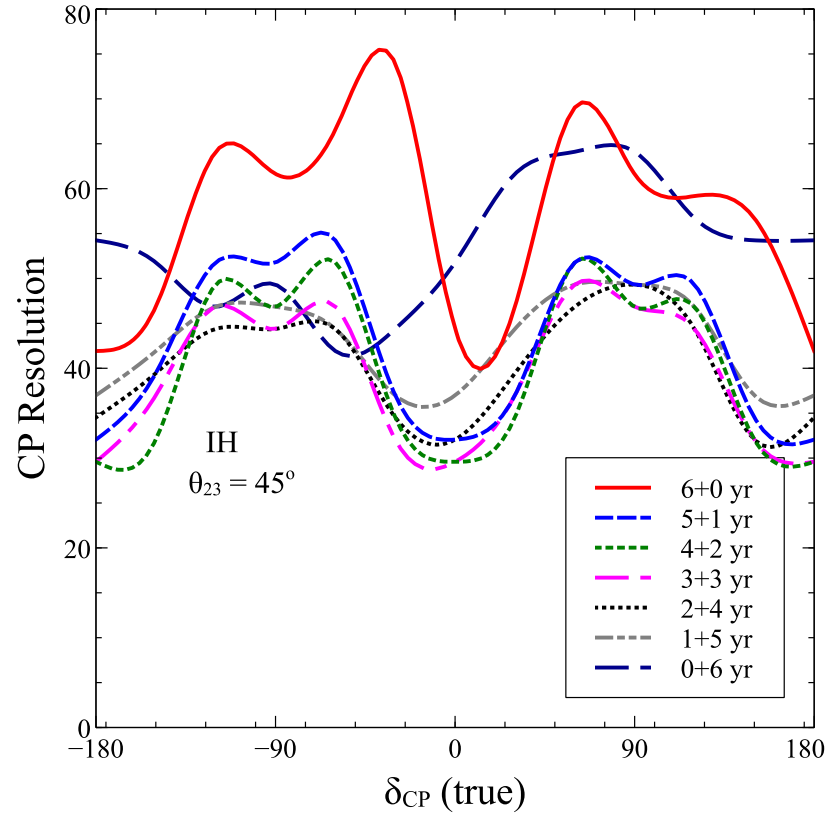

Fig. $12 \mathrm{CP}$ resolution sensitivity in $\mathrm{IH}$ as a function of $\delta_{C P}$ (true) for $\theta_{23}=45^{\circ}$. This is presented for four different neutrino-antineutrino ratios

tain CPV discovery sensitivity as a function of background for different values of systematics. This we have done taking equal ratio of neutrinos and antineutrinos. The true value of $\delta_{C P}$ is $-90^{\circ}$. Similar to the NH case, there is no effect of systematics and background to achieve a sensitivity of $1 \sigma$ as it requires around 1 year for all the three curves. But unlike $\mathrm{NH}$, the effect of systematics is prominent in IH to achieve a sensitivity of $2 \sigma$. For the most conservative case (i.e., $10 \%$ systematic uncertainty), time required to gain $2 \sigma$ sensitivity rises from 4 to 6.5 years when background increases from 5 to $20 \%$. It is also possible to achieve a $3 \sigma$ sensitivity within 10 year of running if the background is less than $7.5 \%$ and systematics in appearance signal channel is absent.

Finally, in Fig. 12 we have given the CP resolution capability in IH for $\theta_{23}=45^{\circ}$ taking different neutrino and antineutrino combinations for a total run-time of 6 years. From the figure we see that the best sensitivity is obtained for $3+3$ years combination and a value of $\delta_{C P}$ (true) $=-90^{\circ}, 0^{\circ}$ and $+90^{\circ}$ can be measured within $30^{\circ}$ of uncertainty.

\section{Comparison of $\mathrm{P} 2 \mathrm{O}$ with other future long-baseline experiments}

In this section we compare the sensitivity of the $\mathrm{P} 2 \mathrm{O}$ experiment with the other future long-baseline experiments which are DUNE, T2HK and T2HKK.

In Fig. 13 we compare the hierarchy, octant and $\mathrm{CP}$ violation sensitivity of the above mentioned experiments in the left, middle and right panel, respectively, for $\mathrm{NH}$. We have taken a total run time of 10 years for all the four experiments. As described in the letter of intents, for DUNE (T2HK/T2HKK) we have taken the 1:1 (1:3) ratio for neutrino and antineutrino run. For $\mathrm{P} 2 \mathrm{O}$ we have taken the most optimized $8+2$ years configuration with two choices of background i.e., 20\% (blue curve) and 5\% (black curve). The choices of true values are mentioned in the figures. For hierarchy sensitivity we see that $\mathrm{P} 2 \mathrm{O}$ wins over all the other experiments even with $20 \%$ background. But for octant, $\mathrm{P} 2 \mathrm{O}$ has the worst sensitivity among the given experiments. However we checked that with $5 \%$ background and $1 \%$ systematic error, the sensitivity of $\mathrm{P} 2 \mathrm{O}$ is comparable to T2HK, for $\theta_{23}$ values closer to maximal. For CPV, P2O and T2HK have comparable sensitivity for $\delta_{C P}=+90^{\circ}$ for $20 \%$ background
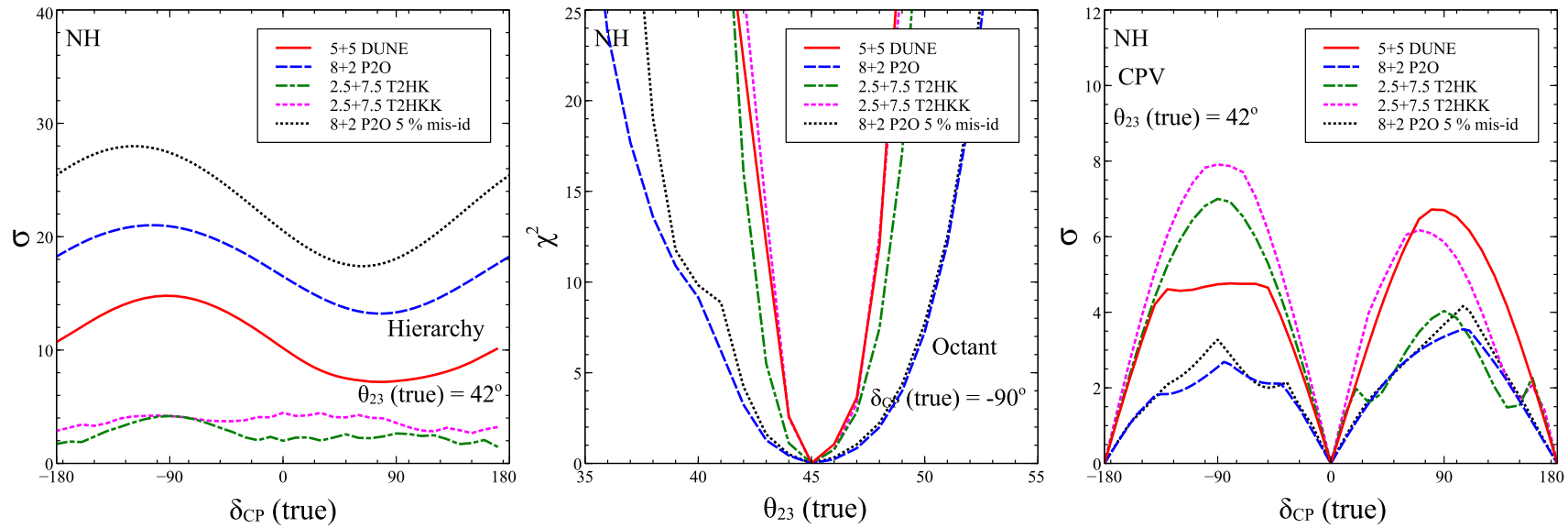

Fig. 13 Comparison of the expected sensitivity of P2O, DUNE, T2HK and T2HKK to discover neutrino mass hierarchy (left panel), octant of $\theta_{23}$ (middle panel) and CPV (right panel). For hierarchy and CPV sensitivity the true value of $\theta_{23}$ is $42^{\circ}$ and for octant sensitivity the true value of $\delta_{C} P$ is $-90^{\circ}$ 
in $\mathrm{P} 2 \mathrm{O}$, while $\mathrm{P} 2 \mathrm{O}$ is better than T2HK for $5 \%$ background. On the other hand, for $\delta_{C P}=-90^{\circ}, \mathrm{P} 2 \mathrm{O}$ has the worst sensitivity for both the choices of background.

\section{Conclusion}

There is a proposal (P2O) to upgrade the Protvino accelerator complex with an increasing beam power of $450 \mathrm{~kW}$ and use it to produce and send a neutrino beam to the ORCA detector in the Mediterranean sea at a distance of $2588 \mathrm{~km}$. The beam will be peaked at about $5 \mathrm{GeV}$ to produce at oscillation maximum at ORCA. The preliminary sensitivity reach of this experiment to neutrino mass hierarchy and $\mathrm{CP}$ violation has been presented by the $\mathrm{P} 2 \mathrm{O}$ collaboration for 3 years of running of the experiment in the neutrino channel. In this paper, we have performed a detailed sensitivity study of $\mathrm{P} 2 \mathrm{O}$ for all three neutrino physics parameters measurable at longbaseline experiments - neutrino mass hierarchy, $\mathrm{CP}$ violation and octant of $\theta_{23}$. The optimisation is done with respect to (i) neutrino vs antineutrino run-time, (ii) detector systematic uncertainties, (iii) total running time of the experiment and (iv) backgrounds coming from mis-id in the appearance channel.

We started by matching our simulated number of events as well a $\chi^{2}$ with the results presented by the $\mathrm{P} 2 \mathrm{O}$ collaboration [19] for 3 years run of the experiment in the neutrino channel and for $\mathrm{NH}$ true. We next calculated the corresponding events for the antineutrinos using fluxes obtained from the P2O collaboration, using the same simulation parameters as for the neutrino channel. We assumed a total run time of 6 years for the experiment and varied the neutrino vs antineutrino run time ratio for mass hierarchy, $\mathrm{CP}$ violation and octant of $\theta_{23}$. We showed that for mass hierarchy measurement, the sensitivity of P2O is always very high for both hierarchies. While the sensitivity does change with neutrino-antineutrino fraction, systematics and backgrounds, especially for $\mathrm{NH}$ true. The significance with which mass hierarchy can be measured remains high for both $\mathrm{NH}$ and $\mathrm{IH}$ true.

The situation with $\mathrm{CP}$ violation and octant of $\theta_{23}$ determination is more complicated for $\mathrm{P} 2 \mathrm{O}$. We found that the significance of $\mathrm{CP}$ violation measurement for the baseline design of the experiment is not as high as that for DUNE and T2HK. For the current best-fit of $\delta_{C P}=-90^{\circ}$ and $\theta_{23}$ (true) $=42^{\circ}$, it can be measured at $2 \sigma$ for $5+1$ years of running of the experiment with $20 \%$ mis-id background. We showed that this could be increased to $3 \sigma$ if the background is reduced to to $5 \%$ and run time increased to $8+2$ years. For octant the reach of $\mathrm{P} 2 \mathrm{O}$ baseline design is expected to be less than $2 \sigma$ at $\theta_{23}$ (true) $=42^{\circ}$ and $\delta_{C P}=-90^{\circ}$ with a background of $20 \%$. We showed that if the mis-id is controlled within $5 \%$, then octant can be measured with $\chi^{2}=5$ for $8+2$ years run of the experiment. Finally, we made a comparative study of P2O along with DUNE, T2HK and T2HKK and showed that $\mathrm{P} 2 \mathrm{O}$ is expected to give the best sensitivity to neutrino mass hierarchy owing to its long baseline which is close to being bi-magic. In terms of $\mathrm{CP}$ violation and octant of $\theta_{23}$ discovery, even though $\mathrm{P} 2 \mathrm{O}$ baseline configuration appears to have a weaker sensitivity compared to DUNE, T2HK and T2HKK, we showed what is needed in order for P2O to be competitive with other long-baseline proposals.

In conclusion, $\mathrm{P} 2 \mathrm{O}$ has the best sensitivity to neutrino mass hierarchy. For octant of $\theta_{23}$ and $\mathrm{CP}$ violation discovery it can be competitive if the experimental collaboration is able to control mis-id and systematics, and run the experiment for $8+2$ years in neutrino and antineutrino mode.

Note that there is a propsal for a super-ORCA detector with approximately 10 times more densely instrumented version of ORCA with lower detection threshold and improved event reconstruction capabilities [36]. It was shown that with this upgraded detector, $\delta_{C P}=0^{\circ}$ and $180^{\circ}$ can be distinguished with $5 \sigma$ confidence level and $60 \%(70 \%)$ of $\delta_{C P}$ values can be disfavoured by more than $2 \sigma$ confidence level for true $\delta_{C P}=0^{\circ}, 180^{\circ}\left( \pm 90^{\circ}\right)$.

Acknowledgements The authors would like to thank Prof. Dmitry Zaborov and Prof. Anatoly Sokolov of the P2O collaboration for providing the fluxes and relevant information regarding the $\mathrm{P} 2 \mathrm{O}$ experiment. The authors would like to thank the Department of Atomic Energy (DAE) Neutrino Project of Harish-Chandra Research Institute. This project has received funding from the European Union's Horizon 2020 research and innovation programme InvisiblesPlus RISE under the Marie Sklodowska-Curie Grant agreement No 690575. This project has received funding from the European Union's Horizon 2020 research and innovation programme Elusives ITN under the Marie SklodowskaCurie Grant agreement no 674896.

Data Availability Statement This manuscript has associated data in a data repository. [Authors' comment: There was some mistake while submission. The data is not stored in any data repository.]

Open Access This article is distributed under the terms of the Creative Commons Attribution 4.0 International License (http://creativecomm ons.org/licenses/by/4.0/), which permits unrestricted use, distribution, and reproduction in any medium, provided you give appropriate credit to the original author(s) and the source, provide a link to the Creative Commons license, and indicate if changes were made.

Funded by SCOAP S $^{3}$

\section{References}

1. DUNE, R. Acciarri et al. (2015). arXiv: 1512.06148

2. Hyper-Kamiokande, K. Abe et al., PTEP 2018 (2018) 063 C01. arXiv: 1611.06118

3. S.B. ESSnu, E. Baussan et al., Nucl. Phys. B 885, 127 (2014). arxiv: 1309.7022

4. S.K. Agarwalla, M. Ghosh, S.K. Raut, JHEP 05, 115 (2017). arxiv: 1704.06116

5. M. Ghosh, O. Yasuda, Phys. Rev. D 96, 013001 (2017). arxiv: 1702.06482

6. S. Fukasawa, M. Ghosh, O. Yasuda, Nucl. Phys. B 918, 337 (2017). arxiv: 1607.03758 
7. N. Nath, M. Ghosh, S. Goswami, Nucl. Phys. B 913, 381 (2016). arxiv: 1511.07496

8. M. Ghosh, S. Goswami, S.K. Raut, Eur. Phys. J. C 76, 114 (2016). arxiv: 1412.1744

9. K. Chakraborty, K.N. Deepthi, S. Goswami, Nucl. Phys. B 937, 303 (2018). arxiv:1711.11107

10. S.K. Raut, Phys. Rev. D 96, 075029 (2017). arxiv: 1703.07136

11. V. Barger et al., Int. J. Mod. Phys. A 31, 1650020 (2016). arxiv: 1405.1054

12. V. Barger et al., Phys. Rev. D 89, 011302 (2014). arxiv:1307.2519

13. S.K. Agarwalla, S. Choubey, S. Prakash, JHEP 12, 020 (2014). arxiv: 1406.2219

14. S.S. Chatterjee, P. Pasquini, J.W.F. Valle, Phys. Rev. D 96, 011303 (2017). arxiv: 1703.03435

15. S.S. Chatterjee, P. Pasquini, J.W.F. Valle, Phys. Lett. B 771, 524 (2017). arxiv: 1702.03160

16. M. Blennow, P. Coloma, E. Fernandez-Martinez, JHEP 03, 005 (2015). arxiv:1407.3274

17. P. Ballett et al., Phys. Rev. D 96, 033003 (2017). arxiv:1612.07275

18. J. Evslin, S.F. Ge, K. Hagiwara, JHEP 02, 137 (2016). arXiv: 1506.05023

19. Scientific potential of a neutrino beam from Protvino to ORCA (P2O), D. Zaborov, 2017, Talk given at Neutrino GDR meeting Paris, France, Nov 20-21, 2017

20. J. Brunner (2013). arXiv:1304.6230

21. KM3NeT, D. Zaborov (2018) 18th Lomonosov Conference on Elementary Particle Physics Moscow, Russia, August 24-30, 2017. arXiv: 1803.08017
22. KM3Net, S. Adrian-Martinez et al., J. Phys. G 43084001 (2016). arXiv: 1601.07459

23. A.V. Akindinov et al., (2019). arXiv: 1902.06083

24. A. Zaitsev (2018) Talk given at Very Large Volume Neutrino Telescopes, 2-4 October 2018, Dubna Russia

25. A. Dighe, S. Goswami, S. Ray, Phys. Rev. Lett. 105, 261802 (2010). arXiv: 1009.1093

26. S.K. Raut, R.S. Singh, S.U. Sankar, Phys. Lett. B 696, 227 (2011). arXiv:0908.3741

27. P. Huber, M. Lindner, W. Winter, Comput. Phys. Commun. 167, 195 (2005). hep-ph/0407333

28. I. Esteban et al. (2018). arXiv:1811.05487

29. P.F. de Salas et al., Phys. Lett. B 782, 633 (2018). arXiv: 1708.01186

30. F. Capozzi et al., Prog. Part. Nucl. Phys. 102, 48 (2018). arXiv:1804.09678

31. S. Prakash, S.K. Raut, S.U. Sankar, Phys. Rev. D 86, 033012 (2012). arXiv:1201.6485

32. M. Ghosh et al., Phys. Rev. D 93, 013013 (2016). arXiv:1504.06283

33. M. Ghosh, Phys. Rev. D 93, 073003 (2016). arXiv:1512.02226

34. S. Prakash, U. Rahaman, S.U. Sankar, JHEP 07, 070 (2014). arXiv: 1306.4125

35. S.K. Agarwalla, S. Prakash, S.U. Sankar, JHEP 07, 131 (2013). arXiv: 1301.2574

36. J. Hofestädt (2018) Psoter given at Neutrino 2018, Heidelberg, June 4-9. https://zenodo.org/record/1292936 